\title{
Developments in Modeling the Galactic Magnetic Field
}

\author{
Glennys R. Farrar* \\ Center for Cosmology and Particle Physics, New York University \\ E-mail: gf25 @ nyu. edu \\ Michael Unger \\ Karlsruhe Institute of Technology \\ E-mail: michael.ungerakit.edu
}

\begin{abstract}
We present an improved 3D model of the strength and structure of the Galactic random field. The analysis uses the synchrotron intensity as measured by Planck, WMAP and Haslam et al. and the observed fluctuations of the rotation measures of extragalactic radio sources. As part of this modeling effort, we improve the separation of the various components of Galactic emission, the determination of the 3D cosmic ray electron density and spectrum, and the thermal electron distribution. This combined information allows us to constrain both the strength and coherence length of the random field. We also report developments in modeling the large scale coherent field of the Galaxy.
\end{abstract}

36th International Cosmic Ray Conference -ICRC2019-

July 24th - August 1st, 2019

Madison, WI, U.S.A.

\footnotetext{
* Speaker.

${ }^{\dagger}$ A footnote may follow.
} 
This contribution for the Proceedings will be completed after the presentation. For related earlier work see $[1,2,3,4,5,6,7,8]$.

\section{References}

[1] R. Jansson and G. R. Farrar, A New Model of the Galactic Magnetic Field, ApJ 757 (2012) 14.

[2] R. Jansson and G. R. Farrar, The Galactic Magnetic Field, Astrophys. J. 761 (2012) L11.

[3] M. Unger and G. Farrar, Progress in the Global Modeling of the Galactic Magnetic Field, EPJ Web Conf. 210 (2019) 04005, [arXiv: 1901 . 04720 ].

[4] M. Unger and G. R. Farrar, Uncertainties in the Magnetic Field of the Milky Way, in Proceedings, 35th International Cosmic Ray Conference (ICRC 2017) 558, 2017. arXiv: 1707.02339.

[5] G. R. Farrar, N. Awal, D. Khurana, and M. Sutherland, The Galactic Magnetic Field and UHECR Optics, in Proceedings, 34th International Cosmic Ray Conference (ICRC 2015), 2015. arXiv:1508.04530.

[6] G. R. Farrar and M. S. Sutherland, Deflections of UHECRs in the Galactic magnetic field, JCAP 1905 (2019), no. 05 004, [arXiv:1711.02730].

[7] J. Kleimann, T. Schorlepp, L. Merten, and J. Becker Tjus, Solenoidal Improvements for the JF12 Galactic Magnetic Field Model, "Astrophys. J." 877 (Jun, 2019) 76, [arXiv: 1809.07528 ].

[8] G. R. Farrar, The Galactic magnetic field and ultrahigh-energy cosmic ray deflections, Comptes Rendus Physique 15 (Apr., 2014) 339-348, [arXiv: 1405.3680 ]. 